\title{
Watermarking in the Space/Spatial-Frequency Domain Using Two-Dimensional Radon-Wigner Distribution
}

\author{
Srdjan Stanković, Member, IEEE, Igor Djurović, Student Member, IEEE, and Ioannis Pitas, Senior Member, IEEE
}

\begin{abstract}
A two-dimensional (2-D) signal with a variable spatial frequency is proposed as a watermark in the spatial domain. This watermark is characterized by a linear frequency change. It can be efficiently detected by using 2-D space/spatial-frequency distributions. The projections of the 2-D Wigner distribution- the 2-D Radon-Wigner distribution, are used in order to emphasize the watermark detection process. The watermark robustness with respect to some very important image processing attacks, such as for example, the translation, rotation, cropping, JPEG compression, and filtering, is demonstrated and tested by using Stirmark 3.1.
\end{abstract}

\section{INTRODUCTION}

D IGITAL watermarking is the area with a very intensive development in the last few years [1]-[7]. It can provide an image copyright protection, i.e., identification of its owner and authorized distributor [1]. The fundamental description of common watermarking techniques is given in the Cox's paper [4]. The most commonly used ways for watermarking are embedding in the spatial domain, or in the transformation (frequency) domain. In both group of methods it is desirable to ensure that a watermark satisfies the following important properties:

1) perceptual invisibility;

2) robustness to the various image processing algorithms, such as common geometric distortions (rotation, translation, cropping), resampling, filtering and compression;

3) ability of watermark detection by copyright owner without the use of the original image.

The frequently used watermarking techniques in the spatial domain are based on the concept of mixing systems [8]-[10]. Watermarking in the spatial-frequency (transformation) domain is commonly performed by embedding a spread-spectrum sequence in the Fourier or DCT domain coefficients [5], [7], [11], [12].

In this paper we propose a watermarking in the joint space/spatial-frequency domain. Two-dimensional (2-D) chirp signals are used as watermarks. This type of signals is resistant to all stationary filtering methods. It also exhibits geometrical symmetry. The Wigner distribution produces an ideal space/spatial-frequency representation for this type of signals [13]-[16]. In order to emphasize the watermark with respect

Manuscript received May 13, 1999; revised December 15, 2000. The work of Prof. Srdjan Stanković was supported by Alexander von Humboldt foundation. The associate editor coordinating the review of this manuscript and approving it for publication was Dr. Naohisa Ohta.

S. Stanković and I. Djurović are with the Electrical Engineering Department, University of Montenegro, Podgorica, Montenegro, Yugoslavia (e-mail: srdjan@cg.ac.yu; igordj@cg.ac.yu).

I. Pitas is with the Department of Informatics, Aristotle Universtity, Thessaloniki, Greece (e-mail: pitas@zeus.csd.auth.gr).

Publisher Item Identifier S 1057-7149(01)02476-9. to the Wigner distribution of the original image, we shall not use the Wigner distribution itself, but its projections (the 2-D Radon-Wigner distribution). In this way the watermark projection maximum will dominate over the values of the Wigner distribution projections of the original image. The chirp signal, after applying linear (affine) geometrical transformations [11], [21]-[23], changes only its position in space/spatial-frequency domain. This is the reason for using two chirp pulses as a watermark, with their relative position being the key for watermark detection. The proposed algorithm falls into the semi-private watermarking schemes, where the embedded watermark is necessary for watermark detection [27].

Here, we present two watermarking techniques. The first one consists in embedding two chirp pulses in an image and detecting the watermark by using the Radon-Wigner distribution. The secret information (the key) is hidden in the relative position of chirp pulses. The second technique is proposed as an extension of the previous one. Multiple chirp pulses with pseudorandom amplitudes are embedded. Detection is performed by using the standard correlation technique [5]. The key consists of amplitudes and positions of the chirp signals. These two techniques could be combined by using the first one as a template, and the second one as a watermark [21]-[23].

The proposed watermarking technique is tested against numerous attacks. Stirmark 3.1 program is used for attacks generation [24]-[26]. Details on fair benchmark of image watermarking systems can be found in [27]. The theory is illustrated on examples, where the watermark detection is performed in the cases of simultaneous rotation, translation and cropping, JPEG compression, and various filtered image forms.

The paper is organized as follows. After an introduction into the 2-D Wigner distribution, Section II, the watermarking detection by using the Radon-Wigner distribution is analyzed in Section III. Multiple linear frequency modulated signals are used as a watermark in Section IV. Numerous examples, illustrating the presented approach, are given in Section V.

\section{THEORETICAL BACKGROUND}

The Wigner distribution of the image $I(x, y)$, is defined as

$$
\begin{aligned}
\operatorname{WD} & \left(x, y, \omega_{x}, \omega_{y}\right) \\
= & \int_{-\infty}^{\infty} \int_{-\infty}^{\infty} I(x+\xi / 2, y+\zeta / 2) I^{*}(x-\xi / 2, y-\zeta / 2) \\
& \times e^{-j\left(\omega_{x} \xi+\omega_{y} \zeta\right)} d \xi d \zeta .
\end{aligned}
$$


Its pseudo form is used in practical realizations

$$
\begin{aligned}
\operatorname{WD} & \left(x, y, \omega_{x}, \omega_{y}\right) \\
= & \int_{-\infty}^{\infty} \int_{-\infty}^{\infty} I(x+\xi / 2, y+\zeta / 2) I^{*}(x-\xi / 2, y-\zeta / 2) \\
& \times w(\xi, \zeta) w^{*}(-\xi,-\zeta) e^{-j\left(\omega_{x} \xi+\omega_{y} \zeta\right)} d \xi d \zeta
\end{aligned}
$$

where $w(\xi, \zeta)$ is a 2-D window function. The 2-D chirp signal, used here as a base for watermarking, has the form

$$
\begin{aligned}
\Omega(x, y) & =2 A \cos \left(a x^{2}+b y^{2}+c\right) \\
& =A\left(e^{j\left(a x^{2}+b y^{2}+c\right)}+e^{-j\left(a x^{2}+b y^{2}+c\right)}\right)
\end{aligned}
$$

where $A$ is the watermark amplitude or strength. Note that the Wigner distribution of this signal is highly concentrated

$$
\begin{aligned}
\operatorname{WD} & \left(x, y, \omega_{x}, \omega_{y}\right) \\
= & A^{2} W\left(\omega_{x}-2 a x, \omega_{y}-2 b y\right) \\
& +A^{2} W\left(\omega_{x}+2 a x, \omega_{y}+2 b y\right)+\text { cross-terms }
\end{aligned}
$$

where the Fourier transform of the window, $W\left(\omega_{x}-2 a x, \omega_{y}-\right.$ $2 b y)$, is close to a delta function $\delta\left(\omega_{x}-2 a x, \omega_{y}-2 b y\right)$ for a sufficiently wide window. Since the cross-terms in the Wigner distribution will be eliminated by use of projections, they will be neglected in the sequel.

After a general linear geometrical transformation, signal (3) can be written in the form

$$
\Omega^{\prime}(x, y)=2 A \cos \left(a_{1} x^{2}+a_{2} y^{2}+a_{3} x y+a_{4} x+a_{5} y+a_{6}\right) .
$$

This transformation corresponds to a mapping of centered ellipse into the rotated one, whose center is displaced from the origin. From the point of view of the Wigner distribution concentration on the local frequency, we may say that it is invariant with respect to this transformation. Only the position of the local frequency, i.e., of the distribution concentration, will be changed

$$
\begin{aligned}
\mathrm{WD} & \left(x, y, \omega_{x}, \omega_{y}\right) \\
= & A^{2} W\left(\omega_{x}-2 a_{1} x-a_{3} y-a_{4}, \omega_{y}-2 a_{2} y-a_{3} x-a_{5}\right) \\
& +A^{2} W\left(\omega_{x}+2 a_{1} x+a_{3} y+a_{4}, \omega_{y}+2 a_{2} y+a_{3} x\right. \\
& \left.+a_{5}\right)+ \text { cross-terms. }
\end{aligned}
$$

This means that the described geometrical transformation does not influence the maximal value of the Wigner distribution, which we intend to use for the watermark detection. Thus, the Wigner distribution of the watermark remains close to the delta pulse [13], [14], [28]. The problem of a changed position of the local frequency (distribution concentration) can be overcome by using two chirp pulses (Section III-C), whose relative position is invariant. Although we have a very specific and recognizable function over the entire space under the consideration, its energy could be much smaller than the energy of the Wigner distribution of an image, since the value of $A$ should be drastically smaller than the average image values. For this reason the watermark detection, using only the Wigner distribution, is not reliable enough.

\section{WATERMARK DETECTION BY USING THE RADON-WIGNER DISTRIBUTION}

Obviously, it is necessary to additionally concentrate the energy of the watermark signal $\Omega(x, y)$. To this end we note that the Wigner distribution maxima in (6) are distributed over the linear hyperplanes in the four-dimensional (4-D) space/spatialfrequency domain, $\omega_{x}= \pm 2 a_{1} x \pm a_{3} y \pm a_{4}, \omega_{y}= \pm 2 a_{2} y \pm$ $a_{3} x \pm a_{5}$, while the Wigner distribution of the image is spread in the 4-D space. Therefore, we shall use the 2-D projections of the Wigner distribution of the watermarked image, rather than the Wigner distribution itself. Analyzing the projections of the Wigner distribution of the watermarked image, one can conclude that a complete concentration of the watermark Wigner distribution (sum of the watermark Wigner distribution maxima) will be achieved in the one projection plane, while the image Wigner distribution will still be spread. Thus, in the projection plane of the watermark, its entire energy will be concentrated in one point. This value, even for a weak watermark, could surpass the image energy from this projection, as well as the watermark energies from the other projection planes. In this way, it will be possible to detect a weak watermark (having an amplitude which is much smaller than the average amplitude of the original image), by using the projections of the Wigner distribution. Note that the position of hyperplanes where watermark is detected depends on the parameters characterizing the geometrical modification of the image. That is the reason for using two chirps as a watermark. For the simplicity of presentation we shall continue to consider just one chirp, having in mind that the final results can be directly applied to the case with two or more chirps.

By using the analogy with the one-dimensional (1-D) case [17]-[20], the projection of the 2-D Wigner distribution, i.e., the 2-D Radon-Wigner distribution can be written in the form

$$
\begin{aligned}
& P_{\mathbf{a}}\left(\omega_{x}, \omega_{y}\right) \\
& =\int_{-\infty}^{\infty} \int_{-\infty}^{\infty} \operatorname{WD}\left(x, y, \omega_{x}, \omega_{y}\right) \delta\left(\omega_{x}-2 a_{1} x-a_{3} y-a_{4},\right. \\
& \left.\quad \omega_{y}-2 a_{2} y-a_{3} x-a_{5}\right) d x d y
\end{aligned}
$$

where $\mathbf{a}=\left(a_{1}, a_{2}, a_{3}, a_{4}, a_{5}\right)$ defines a corresponding hyperplane. The projection maxima

$$
M(\mathbf{a})=\max _{\omega_{x}, \omega_{y}} P_{\mathbf{a}}\left(\omega_{x}, \omega_{y}\right)
$$

that will be used for the watermark detection, are functions of the transformation parameters only.

\section{A. Detection Algorithm}

Consider an image $I(x, y)$ with watermark $\Omega(x, y)$

$$
I^{\prime}(x, y)=I(x, y)+\Omega(x, y)
$$

The Radon-Wigner distribution of $I^{\prime}(x, y)$ can be obtained by multiplying the image $I^{\prime}(x, y)$ by

$$
\Omega_{v}(x, y)=e^{-j\left(a_{v} x^{2}+b_{v} y^{2}+c_{v} x y\right)}
$$


where parameters $a_{v}, b_{v}$ and $c_{v}$ define the projection plane. By computing the 2-D Fourier transform of

$$
I^{\prime}(x, y) \Omega_{v}(x, y)=(I(x, y)+\Omega(x, y)) \Omega_{v}(x, y)
$$

the Radon-Wigner distribution is obtained as

$$
\begin{aligned}
P & \left(\omega_{x}, \omega_{y} ; \Omega_{v}\right) \\
& =\left|\mathrm{FT}_{2 \mathrm{D}}\left\{I^{\prime}(x, y) \Omega_{v}(x, y)\right\}\right|^{2} \\
& =\left|\int_{-\infty}^{\infty} \int_{-\infty}^{\infty} I^{\prime}(x, y) \Omega_{v}(x, y) e^{-j\left(x \omega_{x}+y \omega_{y}\right)} d x d y\right|^{2} .
\end{aligned}
$$

In this way, by using different values of parameters $a_{v}, b_{v}$ and $c_{v}$ we get the Radon-Wigner distribution for different projections. By finding the maxima of the Radon-Wigner distribution of the watermarked image

$$
M\left(a_{v}, b_{v}, c_{v}\right)=\max _{\omega_{x}, \omega_{y}} P\left(\omega_{x}, \omega_{y} ; \Omega_{v}\right)
$$

and by comparing it with an assumed reference threshold, we make a decision about the watermark presence. Thus, the existence of a dominant maximum in the projection amounts to the watermark existence in the particular image. The direct search for three parameters $a_{v}, b_{v}$ and $c_{v}$ can be time consuming. One may deal with this problem by using some of the existing algorithms [29], [30]. The time, necessary for watermark detection for an image size of $512 \times 512$, on a PC $300 \mathrm{MHz}$, is between 55 $\mathrm{s}$ and $1 \mathrm{~min}$. That is the price that has to be paid for the blind watermark detection. In cases of known watermark positions with a multiple chirps having random amplitudes this time consuming search problem does not exist (see Section IV).

Note that the range for possible values of parameters $a$ and $b$ is determined by the maximal possible local frequency of a chirp (3). It is related to the image size $N \times N$, and sampling intervals $\Delta x, \Delta y$, as $|a| \leq \pi /\left(2 N \Delta x^{2}\right),|b| \leq \pi /\left(2 N \Delta y^{2}\right)$. If we use the discrete pseudo Wigner distribution ${ }^{1}$ then, in order to avoid aliasing [28], the maximal possible value of these parameters should be divided by a factor of 2 . However, in the Radon-Wigner distribution the oversampling with respect to the sampling theorem rate is not necessary [19], [20].

\section{B. Performance Analysis}

Now, we can pose the question: What is the minimal watermark level amplitude that can be detected in an image? A general answer is very difficult. However, we shall perform a simplified analysis for a rough performance estimation. Let us assume that the image is a white noise signal having $N \times N$ pixels, and that the space window is $N \times N$. The image energy $E_{I}$ is then uniformly spread over the space/spatial-frequency

${ }^{1}$ The discrete Wigner distribution is defined as

$$
\begin{aligned}
\mathrm{WD} & \left(n_{1}, n_{2}, k_{1}, k_{2}\right) \\
= & \sum_{m_{1}=-N / 2}^{N / 2-1} \sum_{m_{2}=-N / 2}^{N / 2-1} I\left(n_{1}+m_{1}, n_{2}+m_{2}\right) \\
& \times I^{*}\left(n_{1}-m_{1}, n_{2}-m_{2}\right) e^{-j \frac{4 \pi}{N}\left(m_{1} k_{1}+m_{2} k_{2}\right)}
\end{aligned}
$$

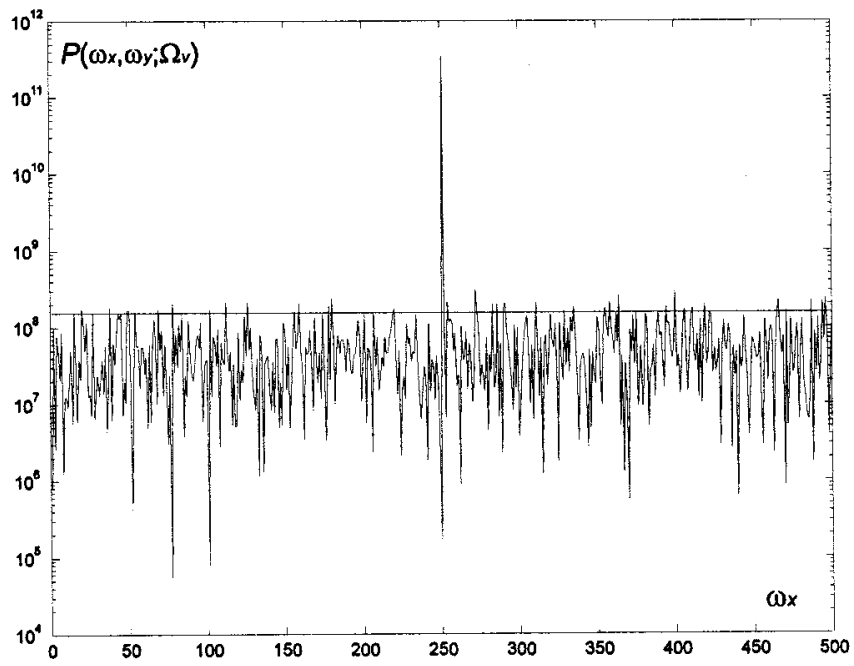

Fig. 1. Illustration of the performance analysis for determination of a detection level of a watermark in the white noisy image. The value of the Radon-Wigner distribution $P\left(\omega_{x}, \omega_{y} ; \Omega_{v}\right)$ as a function of $\omega_{x}$ for the fixed values of $\omega_{y}$ and $\Omega_{v}$, on the line where the watermark is detected.

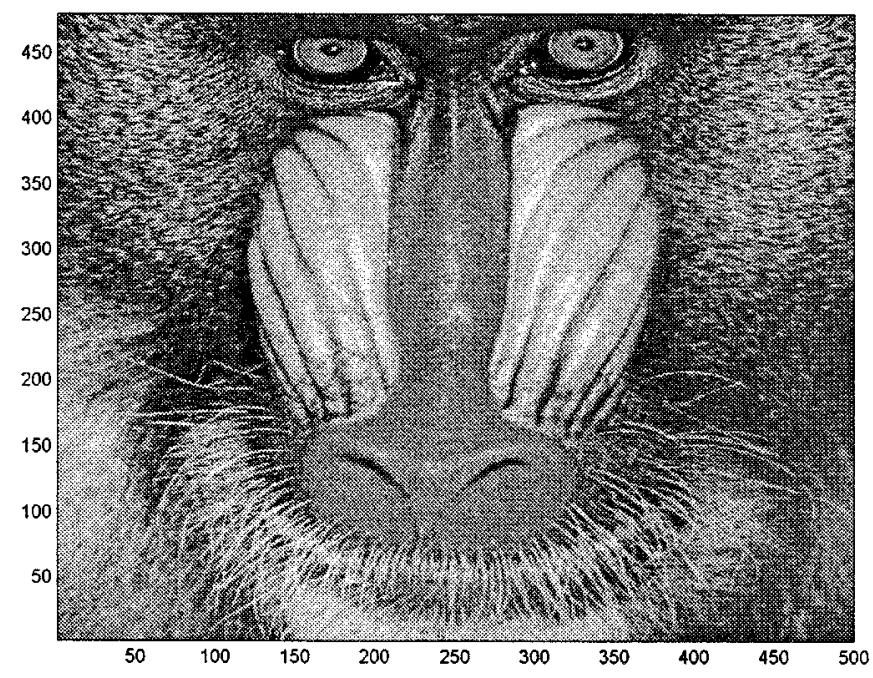

Fig. 2. Original image "Baboon."

domain with energy density proportional to $E_{I} / N^{4}$. The amplitude of the Wigner distribution of the watermark is proportional to $A^{2} N^{2}$. By summing the maxima in the projection plane the maximal expected value of watermark Radon-Wigner distribution is of the order of $A^{2} N^{4}$. The values of the Radon-Wigner distribution of an image is of the order $E_{I} / N^{2}$. The image energy can approximately be represented via the image standard deviation as $E_{I}=A_{I}^{2} N^{2}$ where $A_{I}=\sigma_{I}$. Thus, the ratio of the maxima of the watermark Radon-Wigner distribution and Radon-Wigner distribution of the original image which is relevant for the watermark detection is given by

$$
R=\sqrt{\frac{A^{2} N^{4}}{\frac{E_{I}}{N^{2}}}}=\frac{A}{\sigma_{I}} N^{2} .
$$

It is obvious that the watermark detection performance depends on the ratio $A N^{2} / A_{I}$. Even very small values of $A / A_{I}$ produce a high ratio $R$. Note that one should have an appropriate 

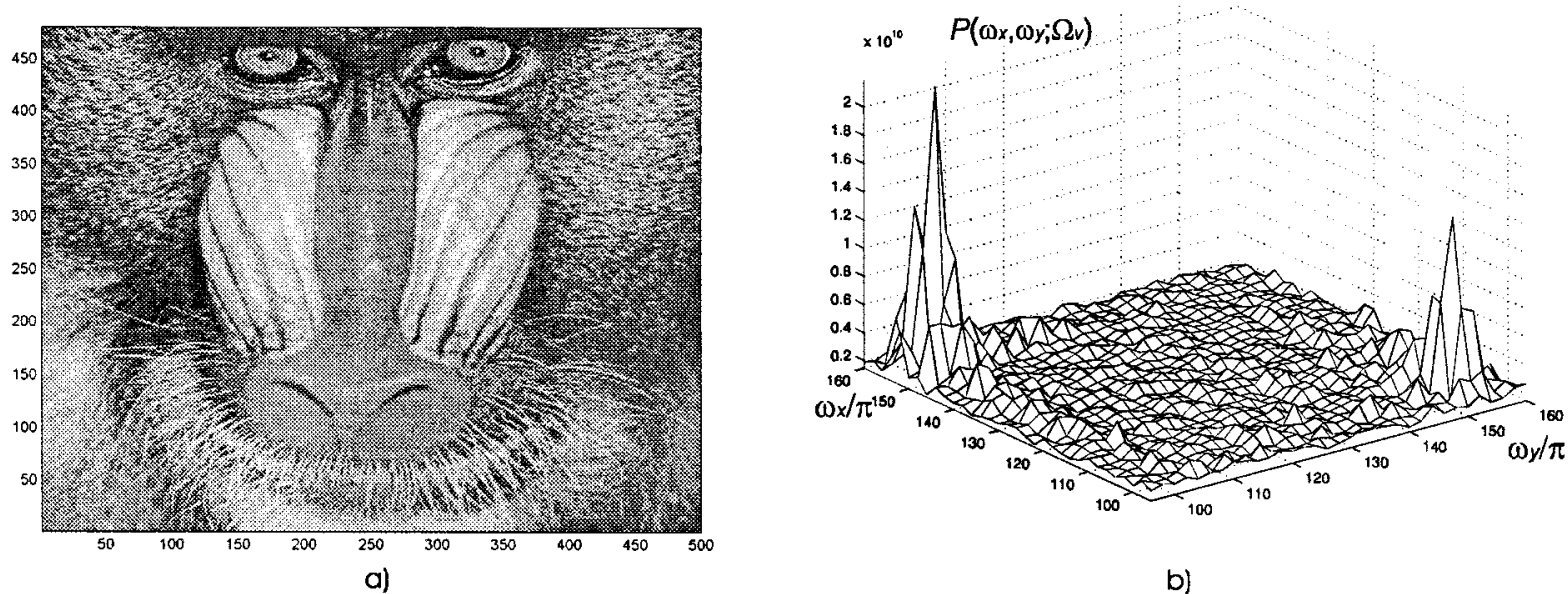

b)

Fig. 3. (a) Watermarked image and (b) maxima of the watermarked image projections and determination of the watermark signal parameters.

margin in a more realistic cases when the image is not white noise. This relation still promises very good detection-visibility ratio. Examples with a real image will be given in Section V. Here, we shall demonstrate the ratio given by (13) on the white noise image, having variance $A_{I}^{2}=\sigma_{I}^{2}=256^{2}$, and watermark with amplitude $A=1.25$. The expected watermark value of $R=1280$ is obtained for $N=512$. That is exactly what we obtained in Fig. 1, where the projection is shown along the line where the watermark maximum is detected.

Taking into consideration that the image energy depends on the particular attack, the detected maximum varies with the image energy. Thus, the threshold is obtained according to

$$
T=\frac{I_{\max }-E_{I}^{\prime} / N^{2}}{E_{I}^{\prime} / N^{2}}
$$

where

$$
\begin{array}{ll}
I_{\max } & \text { dominant maximum value; } \\
E_{I}^{\prime} & \text { energy of the target image; } \\
T & \text { may be taken as } T=1 .
\end{array}
$$

\section{Watermark Robustness}

Since we use as a watermark the 2-D signal with a varying frequency over the entire space, it is resistant to the stationary filtering, i.e., the filtering whose parameters do not change over an image. Also, as previously concluded, the maxima of the Wigner distribution and the maxima of its projections will not be changed after the image translation and rotation. Any reasonable cropping will also not degrade the detection of watermark as long as $R$ remains high. The watermark is resistant to the resampling, as well. However, in this case the position of maxima in projections will be changed. This problem can be overcome by using two or more signals as a watermark. In that case the resampling will change the position of the maxima, but their relative positions will not be changed. The relative position of chirp pulses can be used as a secret key of this watermark. It means that, based on the relative ratio of the positions of maxima, we

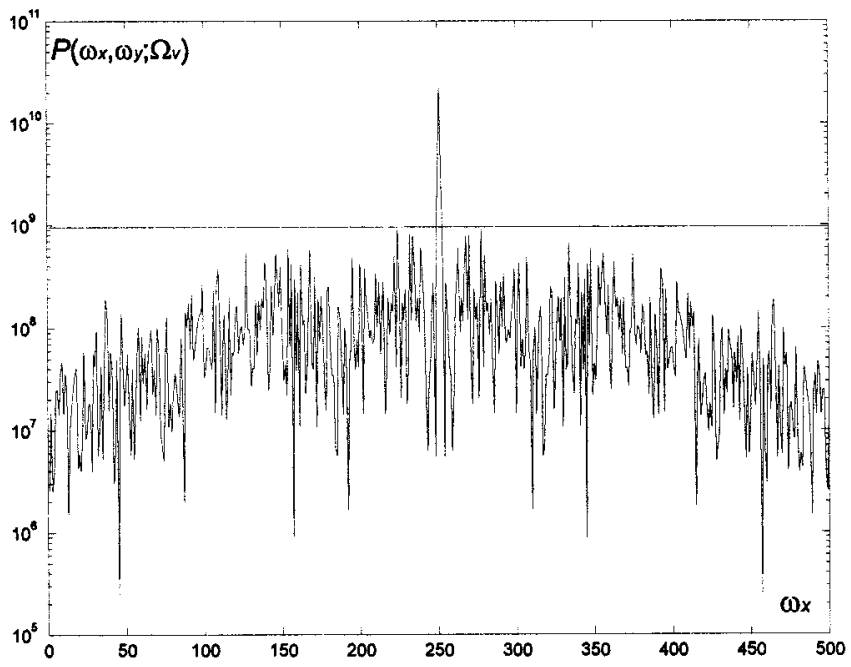

Fig. 4. Radon-Wigner distribution projection along the line where the projection global maximum is detected.

can obtain the information about our watermark after resampling. Also, by using two signals as watermark, we can generate a large number of different watermarks with different relative positions for detection. In this case the watermarked image has the following form:

$$
I^{\prime}(x, y)=I(x, y)+\Omega_{1}(x, y)+\Omega_{2}(x, y)
$$

where

$$
\begin{aligned}
& \Omega_{1}(x, y)=2 A \cos \left(a^{\prime} x^{2}+b^{\prime} y^{2}+c^{\prime}\right) \\
& \Omega_{2}(x, y)=2 A \cos \left(a^{\prime \prime} x^{2}+b^{\prime \prime} y^{2}+c^{\prime \prime}\right) .
\end{aligned}
$$

Here, we should know that there are two chirps as the watermark and detection procedure consists of finding two maxima in the projection planes. This approach will be illustrated in Examples 1 and 2 . 

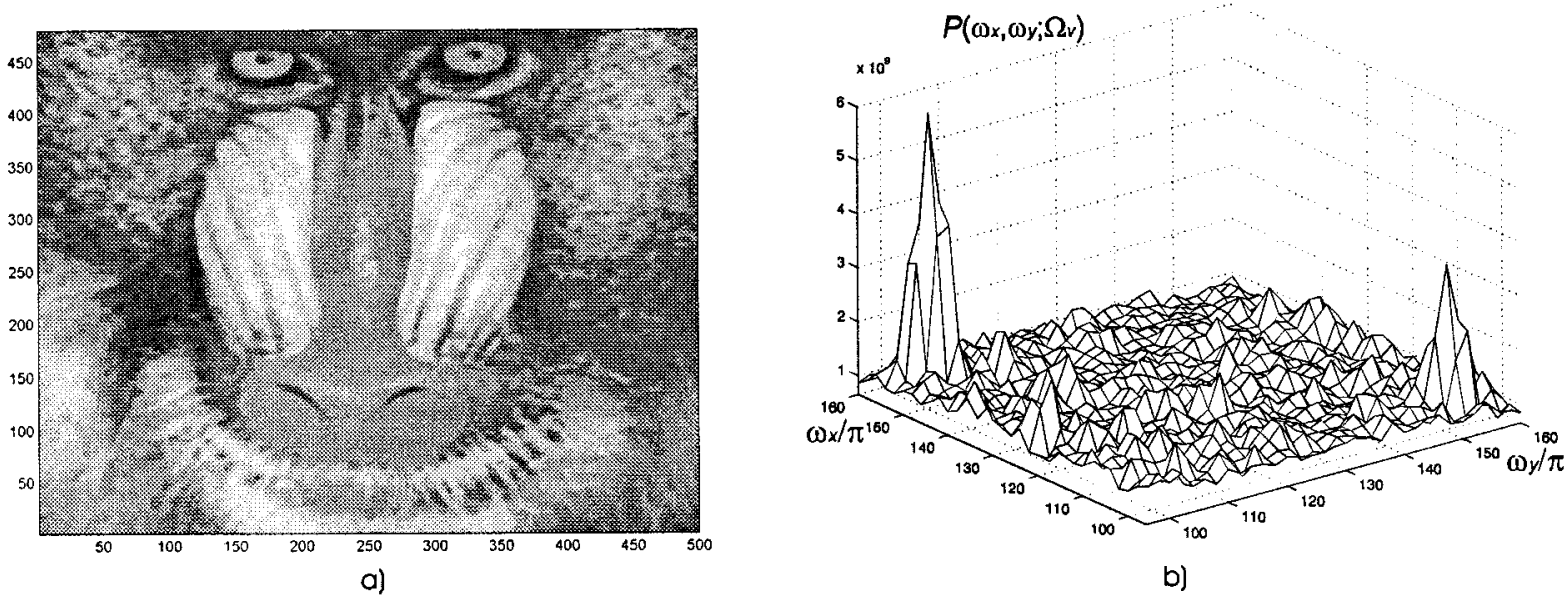

b)

Fig. 5. (a) Watermarked image filtered by a $7 \times 7$ median filter and (b) detection of the watermark parameters in the median filtered image.
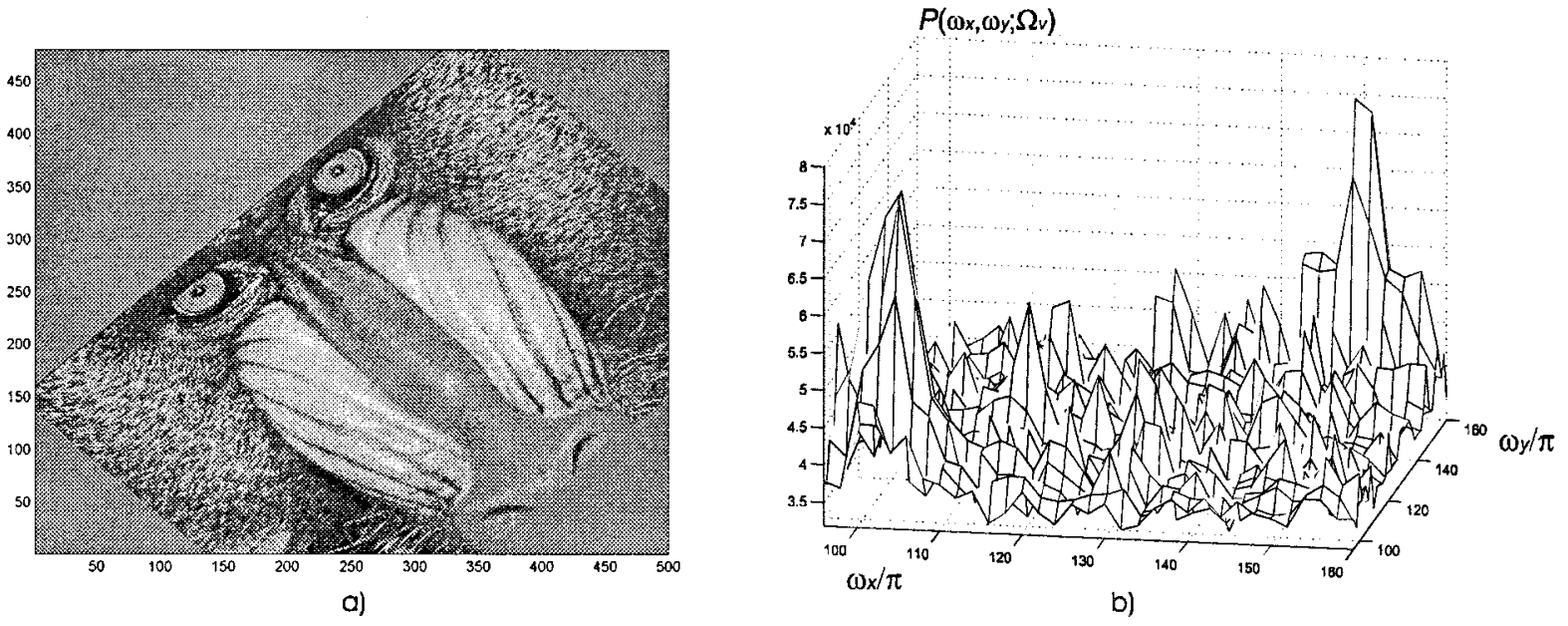

Fig. 6. (a) Watermarked image attacked by a translation, rotation and, cropping and (b) detection of the watermark parameters in the translated, rotated, and cropped image.

\section{Multiple Signals With SMall Amplitudes as A WATERMARK}

The previous concept of watermarking can be extended to the case of watermark signal in the form

$$
\begin{aligned}
& \Omega(x, y) \\
& \quad=\sum_{i=1}^{K} A_{i} \cos \left(a_{i 1} x^{2}+a_{i 2} y^{2}+a_{i 3} x y+a_{i 4} x+a_{i \check{5}} y\right) .
\end{aligned}
$$

Here, in contrast to the previous cases, we shall assume that amplitudes $A_{i}$ are small and randomly chosen and that the number $K$ of chirp signals may be large. Thus, we can generate a very large number of different watermarks. However, the watermark detection will not be possible here without a priori knowledge of the watermark positions, i.e., without knowledge of their projection planes determined by $a_{i 1}, a_{i 2}, a_{i 3}$ and their positions within the specified projection planes defined by $a_{i 4}, a_{i 5}$. It is possible to overcome this problem by combining this concept (randomly

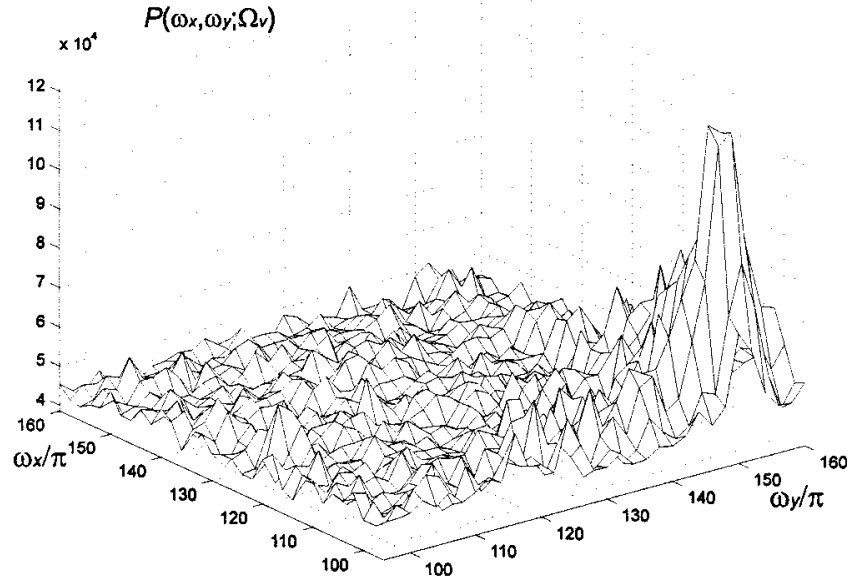

Fig. 7. Detection single watermark embedded in image $\left(a^{\prime}=102 \pi, b^{\prime}=\right.$ $152 \pi$ ) after filtering by a local notch filter.

chosen amplitudes) and the previously described one (deterministic amplitudes in chirp pulses). The detector response can be 


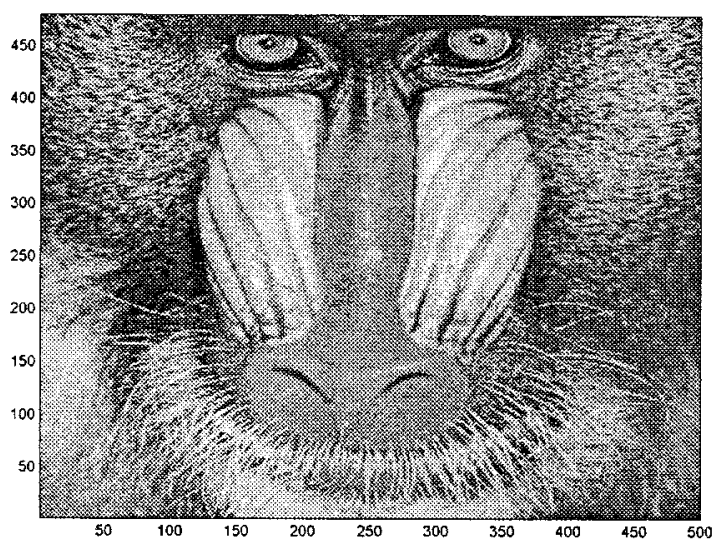

a)

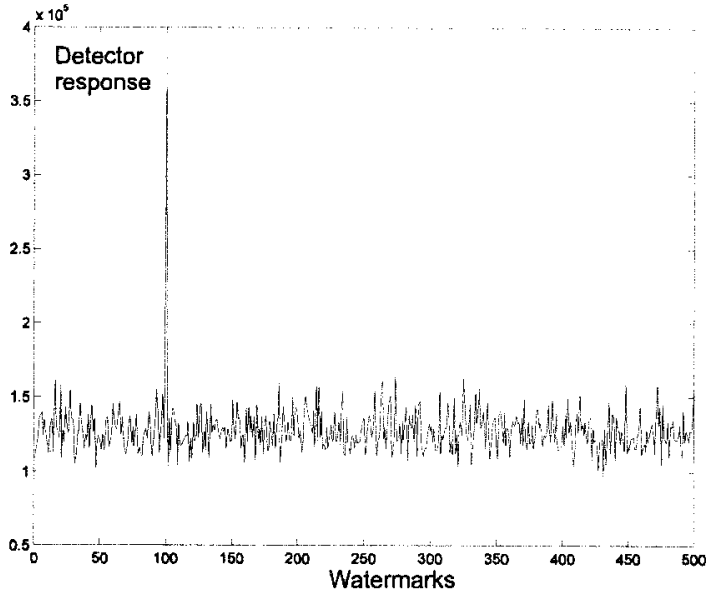

b)

Fig. 8. (a) Image watermarked by a sum of $K=121$ small chirp pulses with randomly generated amplitudes and (b) corresponding detector response.

obtained by using correlation technique

$$
\begin{aligned}
d= & \sum_{i=1}^{M} \mathrm{RWD}_{i} \\
= & \sum_{i=1}^{M} A_{i} \mathrm{FT}_{2 \mathrm{D}}\left[I _ { a } ( x , y ) \operatorname { e x p } \left(-j\left(a_{i 1} x^{2}+a_{i 2} y^{2}\right.\right.\right. \\
& \left.\left.\left.+a_{i 3} x y\right)\right)\right]\left.\right|_{\text {for }\left(\omega_{x}, \omega_{y}\right)=\left(a_{i 4}, a_{i 5}\right)}
\end{aligned}
$$

where $I_{a}(x, y)$ denotes possibly attacked image. This watermarking concept may be considered as a generalized Fourier domain watermarking approach. Fourier domain approach is a special case of (17) with $a_{i 1}=a_{i 2}=a_{i 3}=0$ [11], [21], [31]. The watermarking based on (17) is robust to the attacks either in the Fourier domain or in the spatial domain, since the watermark signal components are well concentrated only in given projection planes which are different for each watermark component. For robustness on geometrical attacks, it is necessary to embed one or two watermark signals with deterministic (higher) amplitude that can be detected by using (11) and (12). The detected changes of parameters of this watermark can be used as a template matching for the detection of (17), [11], [21]-[23]. This will be illustrated on the watermarked images with various attacks (Examples 3 and 4).

\section{EXAMPLES}

Example 1: The image of "Baboon" will be used in this example. The original image with $480 \times 500$ pixels is shown in Fig. 2. Fig. 3(a) represents original image corrupted by watermark (16), where $\left(a^{\prime}=102 \pi, b^{\prime}=152 \pi\right),\left(a^{\prime \prime}=152 \pi, b^{\prime \prime}=\right.$ $102 \pi)$. The peak signal to noise ratio is $P S N R=40.17[\mathrm{~dB}]$, while the signal to noise ratio is $S N R=34.19$ [dB]. We found that in this case $S N R$ is a more appropriate measure for visual image degradation. The watermark amplitude is $A=1.25$, i.e., approximately $1 \%$ of the maximal image amplitude. Note that variation of the amplitude could be considered as well. In the highly textured regions, the amplitude can be higher than in the flatten regions. The watermark level can be determined by using masking systems [5]. Watermark detection is presented

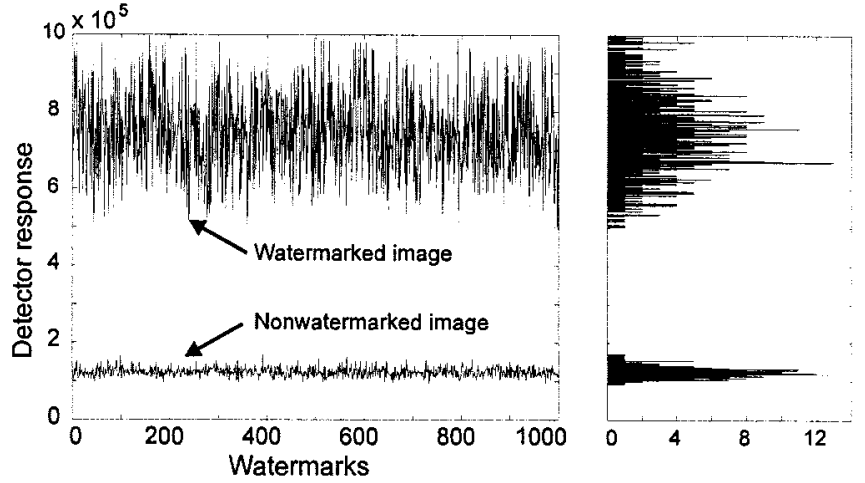

Fig. 9. Statistical check on 1000 realizations of watermarked and nonwatermarked images. Histogram is given on the right hand-side.

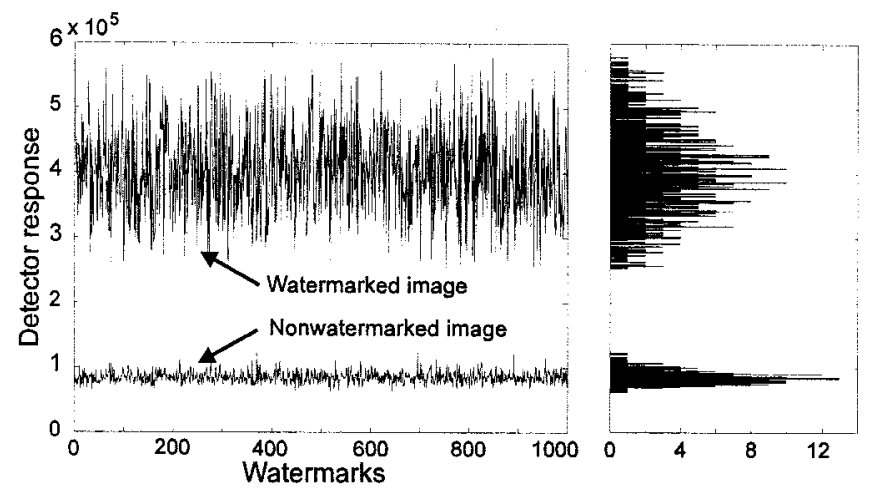

Fig. 10. Statistical check on 1000 realizations of watermarked and nonwatermarked lowpass filtered images.

in Fig. 3(b) where the maxima in various projections are given. Equation (11) is used. It is obvious from Fig. 3(b) that we can easily determine the parameters $\left(a^{\prime}, b^{\prime}\right),\left(a^{\prime \prime}, b^{\prime \prime}\right)$ of the watermark signal. The projection of the Wigner distribution along the line where the maximum $\left(a^{\prime}=102 \pi, b^{\prime}=152 \pi\right)$ is detected is shown in Fig. 4. About $1 \%$ of the low frequency components with extremely high amplitude have been eliminated before the calculation of the projections.

The watermarked image filtered with a $7 \times 7$ median filter is shown in Fig. 5(a), while the watermark detection is presented in 


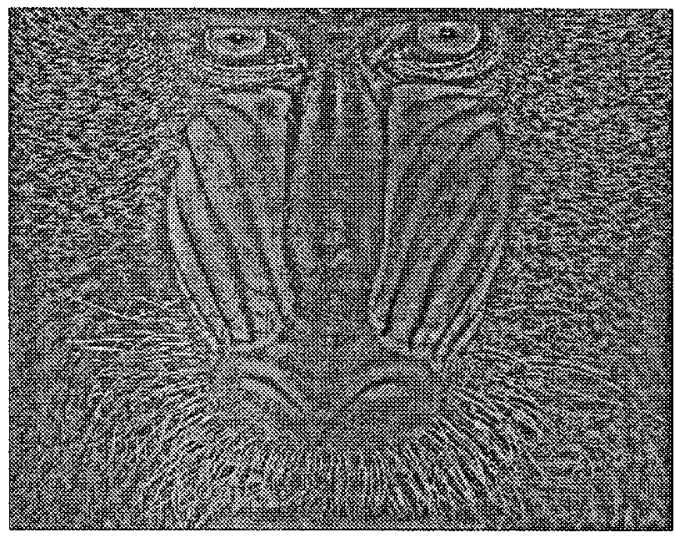

a)

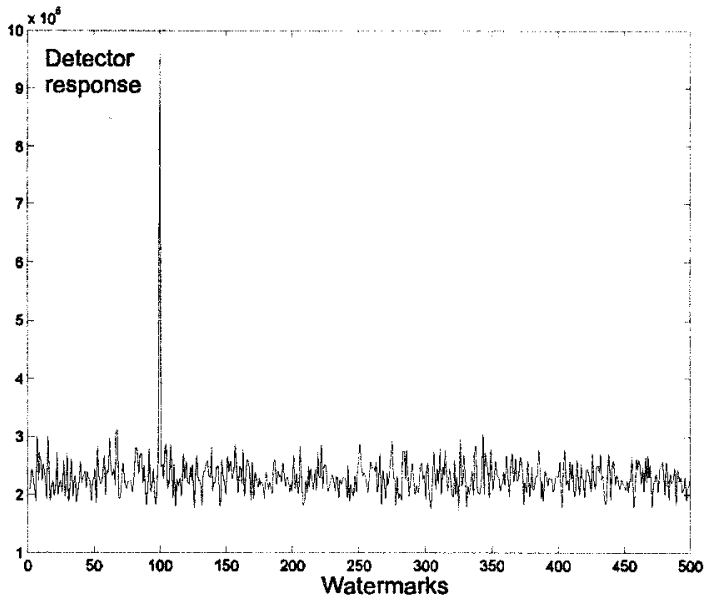

b)

Fig. 11. (a) Highpass filtered image watermarked by a sum of $K=121$ small chirp pulses with randomly generated amplitude and (b) corresponding detector response.

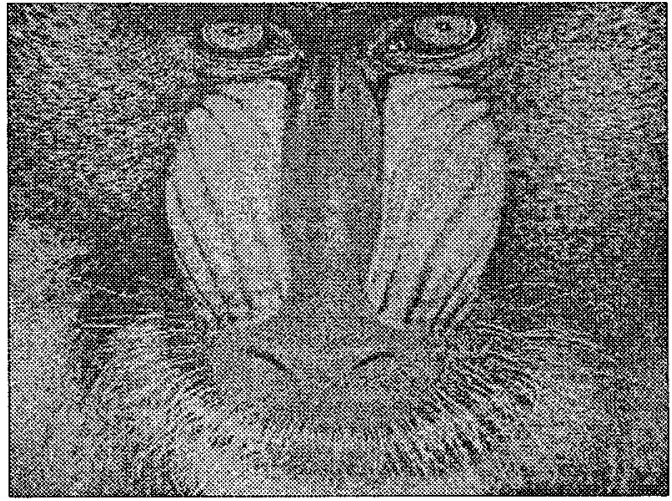

a)

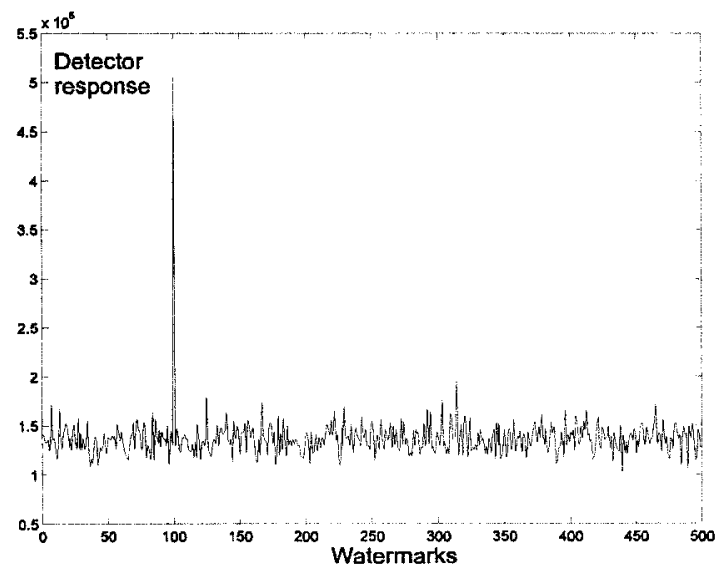

b)

Fig. 12. (a) Watermarked image corrupted with white Gaussian noise and (b) corresponding detector response.

Fig. 5(b). Also, the watermarked image $\left(a^{\prime}=102 \pi, b^{\prime}=102 \pi\right)$, $\left(a^{\prime \prime}=152 \pi, b^{\prime \prime}=152 \pi\right)$ has been attacked by a geometrical distortion consisting of the translation (1/4 of the original image size along the $x$ direction), cropping (1/4 of image has been cropped, along the $x$ direction) and rotation by $45^{\circ}$ (Fig. 6(a)). It is obvious from Fig. 6(b) that the detection of watermark is easily done. Note that this watermarking technique preserves robustness to all linear (affine) geometrical transformations.

Example 2: Here, we shall illustrate that the notch filtering cannot significantly degrade the watermark detection procedure. The watermarked image is filtered by using a notch filter with known local notch frequency positions equal to the watermark chirp local frequencies which can be obtained through the watermark detection procedure. Detection of the watermark, after notch filtering, is shown in Fig. 7. The reason that the notch filtering has not significantly degraded the watermark detection lies in the fact that the watermark signal is not well concentrated in the Fourier domain. The watermark is well concentrated only within its projection plane. The watermark cannot be deleted by using simple subtraction. Namely, the constant phase terms $c^{\prime}$ and $c^{\prime \prime}$, from (16) cannot be found by using the Radon-Wigner distribution, since the image has significant influence on these terms. The problem of chirps deletion would be increased if one used the watermark with varying amplitude.

Note that the previous watermark can be detected by knowing only the watermarking concept without any prior knowledge on the watermark parameters. This blind detection possibility can be avoided by generalizing this concept to the cases with large number of watermark signal components with very small amplitudes and corresponding template matching [11], [21]-[23].

Example 3: An example with $K=121$ component watermarking is illustrated in Fig. 8(a). The watermark amplitudes $A_{i}$ are taken as Gaussian white noise with variance $\sigma_{A}=0.25$. Watermark components are assumed to be positioned on different projection planes. Having in mind that the image content in different projection planes is dispersed, it is not necessary to do watermark adaptation with respect to the image. The detection is illustrated in Fig. 8(b). The response of the detector over 500 randomly created watermarks is given. Only the truly embedded watermark at the index 100 is detected.

The statistical check of this watermarking technique by using 1000 different watermarks and the image from previous exam- 
TABLE I

NuMBER OF DETECTED WATERMARKS OF 26 DIFFERENT EMBEDDED WATERMARKS FOR JPEG COMPRESSION AND ROW/COLUMN REMOVAL

\begin{tabular}{c|c|c|c|c|c}
\hline \hline Attack $\Rightarrow$ & & JPEG & & & Columns and rows removal \\
\hline \hline Image $\Downarrow$ & $10 \%$ & $15 \%$ & $20 \%$ & $\geq 25 \%$ & $1 \times 1,5 \times 1,1 \times 5,5 \times 17,17 \times 5$ \\
\hline \hline Baboon & 26 & 26 & 26 & 26 & 26 \\
\hline \hline Lena & 5 & 7 & 17 & 26 & 26 \\
\hline \hline Fishingboat & 24 & 26 & 26 & 26 & 26 \\
\hline \hline
\end{tabular}

ples is demonstrated in Fig. 9. Fig. 10 gives the statistical results for the lowpass filtered image. We can see that there is a significant gap in the histograms of the watermarked and nonwatermarked images. Accordingly, the decision of the watermark existence is reliable.

The watermarked image filtered by a highpass filter with cutoff frequency equal to $1 / 8$ of the maximal frequency is shown in Fig. 11(a). The corresponding detector response is given in Fig. 11(b). Note that the standard embedding watermark techniques in the frequency domain would not produce satisfactory results for this type of filtering. The watermarked image corrupted with the white Gaussian noise with variance $\sigma=\sqrt{600}$ is shown in Fig. 12(a). Detection of a watermark is shown in Fig. 12(b).

Example 4: Watermarking techniques are usually tested against various robustness criteria. A procedure for fair benchmarking of watermark techniques is presented in [27]. The proposed watermark technique is tested by using Stirmark 3.1 system attacks [24]-[26]. Twenty-six different watermarks are embedded in the standard test images "Baboon," "Lena," and "Fishingboat" $(S N R \approx 34[\mathrm{~dB}]$ in all examples). The watermark is always detected in the cases of median and convolution filtering, general linear geometrical attacks without JPEG compression, as well as with JPEG compression of $90 \%$ quality, including the cases of row and column removal $(1 \times$ $1,1 \times 5,5 \times 1,5 \times 17$, and $17 \times 5$ removal are considered). Table I shows detector response in the case of JPEG compression. The quality levels of $90 \%, 80 \%, 70 \%, 60 \%, 50 \%, 40 \%$, $30 \%, 25 \%, 20 \%, 15 \%$, and $10 \%$ are considered. The watermark is not detected in the case of random geometrical transform (Stirmark attack). We currently consider possibilities of using the time-frequency distributions for generating a watermark that would be resistant to this kind of attacks. Finally, note that the proposed watermark can be detected from a single line (row or column) at the image, since it contains a 1-D linear frequency modulated signal.

\section{CONCLUSION}

The watermark in space/spatial-frequency domain has been presented. The detection of this watermark is performed by using projections of the 2-D Wigner distribution. The robustness of this watermarking scheme to the filtering and geometrical attacks (translation, cropping, rotation) has been demonstrated. The watermarking scheme is tested by using Stirmark 3.1 system. The combination of this watermarking approach with space-varying filtering would offer new possibilities in this area and could be an interesting topic in future research.

\section{ACKNOWLEDGMENT}

The authors are very thankful to the anonymous reviewers for their insightful comments as well as for providing us with the additional references that helped to improve the paper. We are very thankful to Prof. L. Stanković and A. Borş for very helpful suggestions and discussions.

\section{REFERENCES}

[1] G. Voyatzis and I. Pitas, "Protecting digital image copyrights: A framework," IEEE Comput. Graph. Applicat., vol. 19, pp. 18-23, Jan. 1999.

[2] Proc. IEEE, vol. 87, July 1999. Special issue on identification and protection of multimedia information.

[3] Signal Processing, vol. 66, no. 3, 1998. Special issue on watermarking.

[4] I. J. Cox, J. Kilian, F. T. Leighton, and T. Shamoon, "Secure spread spectrum watermarking for multimedia," IEEE Trans. Image Processing, vol. 6, pp. 1673-1687, Dec. 1997.

[5] A. Piva, M. Barni, F. Bartolini, and V. Cappellini, "DCT-based watermark recovering without resorting to the uncorrupted original image," in IEEE Int. Conf. Image Processing'97, Santa Barbara, CA, Oct. 1997, pp. 520-523.

[6] G. Voyatzis, N. Nikolaidis, and I. Pitas, "Digital watermarking: An overview," in Proc. Eur. Signal Processing Conf. EUSIPCO, Rhodes, Greece, Sept. 1998, pp. 9-12.

[7] A. Bors and I. Pitas, "Image watermarking using DCT domain constrain," in IEEE Int. Conf. Image Processing'96, Laussanne, Switzerland, Sept. 1996, pp. 231-234.

[8] N. Nikolaidis and I. Pitas, "Robust image watermarking in the spatial domain," Signal Process., vol. 66, no. 3, pp. 385-403, 1998.

[9] G. Voyatzis and I. Pitas, "Digital image watermarking using mixing systems," Comput. Graph., vol. 22, no. 4, pp. 405-416, 1998.

[10] - "Chaotic watermarks for embedding in the spatial digital image domain," in Proc. IEEE Int. Conf. Image Processing (ICIP'98), Chicago, IL, Oct. 4-7, 1998.

[11] J. J. K. O'Ruanaidh and T. Pun, "Rotation, scale and translation invariant spread spectrum digital image watermarking," Signal Process., vol. 66, pp. 303-318, May 1998.

[12] J. J. K. O'Ruanaidh, W. J. Dowling, and F. M. Bowland, "Phase watermarking of digital image," in Proc. IEEE Int. Conf. Image Processing (ICIP'96), vol. III, Lausanne, Switzerland, Sept. 1996, pp. 239-242.

[13] Y. M. Zhu, F. Peyrin, and R. Goutte, "Equivalence between the twodimensional real and analytic signal Wigner distribution," IEEE Trans. Acoust., Speech, Signal Processing, vol. 37, pp. 1631-1634, Oct. 1989.

[14] S. Stanković, Lj. Stanković, and Z. Uskoković, "On the local frequency, group shift and cross-terms in the multidimensional time-frequency distributions; a method for multidimensional time-frequency analysis," IEEE Trans. Signal Processing, vol. 43, pp. 1719-1725, July 1995.

[15] J. Hormigo and G. Cristobal, "High resolution spectral analysis of images using the pseudo-Wigner distribution," IEEE Trans. Signal Processing, vol. 46, pp. 1757-1763, June 1998.

[16] S. Stanković, I. Djurović, and V. Vuković, "An architecture for the realization of system for space-frequency image analysis," Electron. Lett., vol. 34, no. 23, pp. 2224-2245, Nov. 1998.

[17] B. Ristić and B. Boashash, "Kernel design for time-frequency signal analysis using the Radon transform," IEEE Trans. Signal Processing, vol. 41, pp. 1996-2008, May 1995.

[18] J. C. Wood and D. T. Barry, "Linear signal synthesis using the Radon-Wigner distribution," IEEE Trans. Signal Processing, vol. 42, pp. 2105-2111, Aug. 1994.

[19] _ , "Radon transform of time-frequency distributions for analysis of multicomponent signals," IEEE Trans. Signal Processing, vol. 42, pp. 3166-3177, Nov. 1994.

[20] — "Time-frequency analysis of skeletal muscle and cardiac vibrations," Proc. IEEE, vol. 84, pp. 1281-1294, Sept. 1996.

[21] S. Pereira, J. J. K. ÓRuanaidh, F. Deguillaume, G. Csurka, and T. Pun, "Template based recovery of Fourier-based watermarks using log-polar and log-log maps," in IEEE Multimedia Systems 99, Int. Conf. Multimedia Computing Systems, vol. 1, Florence, Italy, June 7-11, 1999, pp. 870-874. 
[22] S. Pereira and T. Pun, "Fast robust template matching for affine resistant image watermarking," in Int. Workshop Information Hiding, Dresden, Germany, http://cuiwww.unige.ch/ vision/Publications/ThierryPun_publications_refpap.html. available on-line.

[23] G. Csurka, F. Deguillaume, J. J. K. ÓRuanaidh, and T. Pun, “A Bayesian approach to affine transformation resistant image and video watermarking," in IWIH'99, Dresden, Germany, http://cuiwww.unige.ch/ vision/Publications/ThierryPun_publications_refpap.html. available [24] on-line.

[25] F. A. P. Petitcolas, R. J. Anderson, and M. G. Kuhn, "Attacks on copyright marking systems," in IWIH'98, Proceedings, LNCS 1525, ISBN 3-540-65 386-4, D. Aucsmith, Ed.. Portland, Oregon, U.S.A., pp. 219-239. .

[26] F. A. P. Petitcolas and R. J. Anderson, "Evaluation of copyright marking systems," presented at the IEEE Multimedia Systems (ICMCS'99), Florence, Italy, June 7-11, 1999.

[27] M. Kutter and F. A. P. Petitcolas, "A fair benchmark for image watermarking systems," Electronic Imaging'99, Security and Watermarking Contents, vol. 3657, Jan. 25-27, 1999.

[28] A. H. Costa and G. F. Boudreaux-Bartels, "An overview of aliasing errors in discrete-time formulations of time-frequency representations," IEEE Trans. Signal Processing, vol. 47, pp. 1463-1474, May 1999.

[29] A. Papoulis, Signal Analysis. New York: McGraw-Hill, 1977.

[30] L. J. Stanković and I. Djurović, "Relationship between the ambiguity function and fractional Fourier transform," Annales des Telecommunications, vol. 53, no. 3/4, pp. 70-73, Mar./Apr. 1998.

[31] A. Herrigel, J. J. K. ÓRuanaidh, H. Petersen, S. Pereira, and T. Pun, "Secure copyright protection technique for digital images," in Proc. Int. Workshop Information Hiding, Apr. 1998.

Srdjan Stanković (M'94) was born in Montenegro, Yugoslavia, on May 9, 1964. He received the B.S. degree (with honors) in 1988, from the University of Montenegro, the M.S. degree from the University of Zagreb, Croatia, in 1991, and the Ph.D. degree from the University of Montenegro, in 1993, all in electrical engineering.

From 1988 to 1992, he was a Research Assistant with the Aluminum Plant, Podgorica, Montenegro. In 1992, he joined the Electrical Engineering Department, University of Montenegro, where he is currently Associate Professor. His interests are in signal processing and digital electronics. Several of his papers have appeared in leading journals. He published a textbook about electronic devices in Serbo-Croat, and coauthored a monograph on time-frequency signal analysis. During 1999-2000, he was on leave at the Institute of Communications Technology, Darmstadt University of Technology, Germany, supported by the Alexander von Humboldt Foundation.

Dr. Stanković was awarded the Montenegrin Academy of Science and Art Biannual Young Researcher Prize in 1995.
Igor Djurović (S'99) was born in Montenegro, Yugoslavia, in 1971. He received the B.S., M.S., and Ph.D. degrees in electrical engineering from the University of Montenegro, in 1994, 1996, and 2000, respectively.

His current research interests include application of virtual instruments, timefrequency analysis based methods for signal estimation and filtering, fractional Fourier transform applications, image processing, and digital watermarking. He is co-author of three books.

Ioannis Pitas (M'84-SM'94) received the Diploma of Electrical Engineering in 1980 and the Ph.D. degree in electrical engineering in 1985 both from the University of Thessaloniki, Greece. Since 1994, he has been a Professor at the Department of Informatics, University of Thessaloniki. From 1980 to 1993 he served as Scientific Assistant, Lecturer, Assistant Professor, and Associate Professor in the Department of Electrical and Computer Engineering at the same University. He served as a Visiting Research Associate at the University of Toronto, Canada, University of Erlangen-Nuernberg, Germany, Tampere University of Technology, Finland and as Visiting Assistant Professor at the University of Toronto. He was lecturer in short courses for continuing education. His current interests are in the areas of digital image processing, multidimensional signal processing and computer vision. He has published over 300 papers and contributed in 8 books in his area of interest. He is the co-author of the book "Nonlinear Digital Filters: Principles and Applications" (Kluwer, 1990) and author of "Digital Image Processing Algorithms" (Prentice Hall, 1993). He is the editor of the book "Parallel Algorithms and Architectures for Digital Image Processing, Computer Vision and Neural Networks" (Wiley, 1993). Dr. Pitas has been member of the European Community ESPRIT Parallel Action Committee. He has also been an invited speaker and/or member of the program committee of several scientific conferences and workshops. He was Associate Editor of the IEEE Transactions on Circuits and Systems and co-editor of Multidimensional Systems and Signal Processing and he is currently an Associate Editor of the IEEE Transactions on Neural Networks. He was chair of the 1995 IEEE Workshop on Nonlinear Signal and Image Processing (NSIP95). He was technical chair of the 1998 European Signal Processing Conference. He is general chair of IEEE ICIP2001. 